\title{
The effects of embryo culture media on the birthweight of singletons via fresh or frozen-thawed embryo transfer: a large- scale retrospective study
}

Fang $\mathrm{Gu}^{1}$, Mingfen Deng ${ }^{1}$, Jun Gao ${ }^{1}$, Zilian Wang ${ }^{3}$, Chenhui Ding ${ }^{1,2}$, Yanwen $\mathrm{Xu}^{1,2^{*}}$ and Canquan Zhou ${ }^{1,2^{*}}$

\begin{abstract}
Background: Embryo culture media used for IVF treatment might affect fetal growth and thus birthweight of the newborns.

Methods: A retrospective study was conducted in South China using data from 2370 singleton neonates born after IVF/ICSI between 2009 and 2012. Two culture media, i.e., either Vitrolife or SAGE were used as embryo culture media during the study period. Neonates' birthweights were compared between the two embryo culture media groups.

Results: Among the 2370 singletons, 1755 cases came from fresh cleavage embryo transfer while 615 were from frozen-thawed cleavage embryo transfer. Within the fresh embryo transfer newborns, no statistical difference was observed in either birthweight (mean \pm SD: $3196.0 \pm 468.9$ versus $3168.4 \pm 462.0 \mathrm{~g}, p>0.05$ ) or adjusted birthweight controlled for gestational age and gender (z-score mean \pm SD: $0.11 \pm 1.02$ versus $0.11 \pm 0.99 \mathrm{~g}, P>0.05$ ) between the Vitrolife $(n=419)$ and the SAGE group $(n=1336)$. Likewise within frozen embryo transfer neotates, no statistical difference of the birthweight ( $3300.6 \pm 441.3$ vs.3256.0 $\pm 466.7 \mathrm{~g}, P>0.05)$ and adjusted birthweight $(0.30 \pm 0.99 \mathrm{~g}$ versus $0.29 \pm 0.97 \mathrm{~g}, P>0.05)$ was found between the Vitrolife $(n=202)$ and the SAGE group $(n=413)$. The sex ratio [OR1.17, $95 \% \mathrm{Cl}(0.94-1.46) / \mathrm{OR} 1.1,95 \% \mathrm{Cl}(0.78-1.54)]$, rate of small for gestational age [OR1.14, $95 \% \mathrm{Cl}(0.82-1.59) /$ OR1.06, $95 \% \mathrm{Cl}(0.56-2.02)]$ and large for gestational age [OR1.07, $95 \% \mathrm{Cl}(0.64-1.76) / \mathrm{OR} 0.98,95 \% \mathrm{Cl}(0.47-2.02)]$ in fresh and frozen-thawed subgourps are all comparable respectively between the two culture media. No group differences were found in the rate of low birthweight and macosomia. Multiple linear regression analysis demonstrated that maternal weight, gestational age, frozen-thawed embryo transfer and infant gender were significantly related to neonatal birthweight $(P<0.001)$.
\end{abstract}

Conclusions: It appears that embryos cultured in SAGE or Vitrolife media after fresh or frozen-thawed cleavage embryo transfer did not affect neonate's birthweight.

Keywords: IVF, Birthweight, Embryo culture medium, Singleton, Vanishing twin

\footnotetext{
* Correspondence: xuyanwen@live.cn; zhoucanquan@hotmail.com

${ }^{1}$ Department of Obstetrics and Gynecology, Reproductive Medical Center,

The First Affiliated Hospital of SunYat-senUniversity, No 58 Zhongshan Road

2, Yuexiu District, Guangzhou 510080, China

Full list of author information is available at the end of the article
} 


\section{Background}

The Assisted Reproductive Technology (ART) is thought to be associated with an increased risk of adverse perinatal outcomes such as preterm birth, low birth weight, fetal malformation and being born small for gestational age (SGA) or large for gestational age (LGA) [1-5]. Despite the high prevalence of twins and triplets associated with ART which contributes to the increased risk of these adverse outcomes, singletons born after ART also performed worse than their naturally conceived peers [6]. Nowadays, the aspects of ART itself believed to affect perinatal outcomes are ovarian stimulation, laboratory procedures and the culture media [7].

Whether embryo culture media affect fetal growth and birthweight remain inconclusive. Several studies showed that culture media used during IVF treatment could influence intrauterine growth and birthweight of newborns $[8,9]$. In contrast, other research did not confirm such an adverse effect [10-12].

Vitrolife and SAGE medium are the two commonly used embryo culture media in China. They are both sequential culture media for embryo culture with similar components. SAGE is produced in the United States (US) and mainly used in patients in US and Asia, while Vitrolife is more widely used in Europe. Thus it would be interesting to compare the perinatal outcome of these two culture media in the same population under the same laboratory condition. As such, a retrospective study was conducted to investigate the effect of these two culture media, Vitrolife and SAGE, on the birthweight of singleton newborns in a large population conceived from fresh and frozen-thawed embryo transfer.

\section{Methods}

\section{Study design and study population}

This was a retrospective study. Subjects were singletons born alive over 28 weeks of gestation who underwent IVF/ICSI cycles with cleavage embryo transfer between June 2009 and October 2012 in the reproductive medical center of the First Affiliated Hospital of SunYat-sen University. Women applying for blastocyst transfer, preimplantation genetic diagnosis were excluded from the study. The research protocol was approved by Ethic Review Committee of the First Affiliated Hospital of SunYat-sen University and informed consents were obtained prior to data collection. Research nurses contact mothers to obtain information on perinatal outcomes including birthweight, gestational age and gender. Pregnancies lost to follow-up were excluded from data analysis.

\section{Stimulation regimens}

Women underwent controlled ovarian hyperstimulation with a GnRH agonist and antagonist protocol. Ovarian follicle development was monitored based on serum estradiol $\left(E_{2}\right)$ levels and transvaginal ultrasonographic measurements. When at least three follicles reached a mean diameter of $18 \mathrm{~mm}, 250 \mu \mathrm{g}$ recombinant hCG (Serono, Aubonne, Switzerland) was administered $36 \mathrm{~h}$ before ultrasonography-guided oocyte retrieval. Luteal support was initiated on the day after oocyte retrieval using $40 \mathrm{mg}$ of progesterone.

\section{Laboratory protocols}

Two culture media were used: G5 ${ }^{\mathrm{TM}}$ (Vitrolife, Gottenburg, Sweden) and Quinn's advantage medium (SAGE, CA, USA) for embryo culture. The corresponding sera supplemented the media: HSA solution ${ }^{\mathrm{TM}}$ (Vitrolife) and Quinn's advantage SPS (SAGE). Oocytes were placed in either SAGE or Vitrolife after oocyte retrieval. Then IVF/ICSI were performed according to the laboratory's routine insemination procedures. The presence of two pronuclei was observed $16-18 \mathrm{~h}$ after insemination or injection, and the zygotes were then cultured in $25 \mu$ pre-equilibrated cleavage medium droplets (G1and Quinn's advantage cleavage medium). The embryos were cultured in incubators at $37.0{ }^{\circ} \mathrm{C}$ under $6 \% \mathrm{CO}_{2}$ in Vitrolife group and $5 \% \mathrm{CO}_{2}$ in $\mathrm{SAGE}$ group strictly following the product instructions. The morphology of embryos was evaluated $68-72 \mathrm{~h}$ after insemination with respect to cell number, fragmentation and symmetry.

Embryo quality assessment protocol was based on the standard of Istanbul consensus workshop on embryo assessment [13]. The scoring format was cell number, grade and fragmentation. "Good quality" Day-3 embryos were defined as 7-9 equally sized mononucleated blastomeres, with $<10 \%$ fragmentation.

\section{Embroy transfer protocols}

On day 3 after oocyte retrieval, embryo transfer was provided and the number of transfer embryos was determined based on patient's age, number of IVF cycles and embryo quality. Normally, we transferred two Day 3 embryos in patients under 35 years old in their first cycle and transferred three Day3 embryos in other cases. Surplus good quality embryos were cryopreserved through slow-freezing or vitrification.

In frozen embryo transfer (FET) cycles, endometrium preparation protocols including natural cycle and hormone replacement treatment (HRT) cycle were administrated according to the characteristics of patients. The natural cycle preparation protocol included cycle evaluation using ultrasound monitoring of follicular size and endometrial thickness. The day of ovulation was determined by the disappearance of the leading follicle combined with decline of $E_{2}$ and elevation of progesterone level. Embryos were transferred on Day 3 after ovulation. HRT cycles were performed to patients who did not have regular ovulation. Participants started oral administration 
of $4 \mathrm{mg} / \mathrm{d} \mathrm{E}_{2}$ valerate (Progynova, Schering Health Care) for endometrium preparation. An ultrasound assessment was done 12 to 14 days later to assess the uterus as ready for the ET procedure when the endometrium thickness exceeded $8 \mathrm{~mm}$. If not adequate, endometrial priming continued with step-up dose of $E_{2}$ and monitoring scans were undertaken to confirm further endometrial thickening. Participants commenced luteal support via $40 \mathrm{mg}$ progesterone injection for three days before ET.

\section{Criteria for data collection}

Birthweight comparison was our major goal. As gestational age and gender are crucial factors for fetal birthweight [14], we not only compared the absolute birthweight but also the birthweight adjusted for gestational age and gender, which was known as the z-score. The z-score of each individual case was calculated using the following formula: "the weight of the individual child minus the median weight of a reference population of children born at the same gestational age and of the same gender divided by the standard deviation (at the same gestational age and the same gender)" $[15,16]$. The reference population represented the latest available birthweight data for the same province in China [17]. We defined low birthweight (LBW) and macrosomia according to the international description [18]. Small for gestational age (SGA) and large for gestational age (LGA) were defined based on the standards referenced in the book of Chinese Perinatology [19].

\section{Statistical analysis}

All statistical analyses were performed with SPSS 17.0 software. The basic characteristics of the patients were compared using Student's t-tests (continuous variables) and categorical variables were evaluated with $X^{2}$ tests. Multiple linear regression analyses were used to evaluate the association between culture media and birthweight, while controlling for the effects of possible confounding factors, such as fertilization methods (IVF or ICSI), fresh or frozen-thawed embryo transfer, maternal age, maternal weight, gestational age and infant gender.

\section{Results}

\section{Patient and cycle characteristics}

A total of 2370 newborns were studied. Among these, 1755 were babies delivered from fresh embryo transfer with 419 cultured in Vitrolife and 1336 in SAGE; while 615 cases were delivered from frozen cleavage embryo transfer group, with 202 embryos cultured in Vitrolife and 413 cultured in SAGE. Each couple contributed only one pregnancy to this study.

The maternal characteristics are shown in Tables 1 and 2. All patients were Chinese non-smoking women. No significant differences were found between these two media in patients' characteristics, except maternal age in
Table 1 Patients' characteristics and neonatal outcome after fresh cleavage-stage embryo transfer

\begin{tabular}{llll}
\hline & $\begin{array}{l}\text { Vitrolife } \\
(n=419)\end{array}$ & $\begin{array}{l}\text { SAGE } \\
(n=1336)\end{array}$ & $p$ \\
\hline Maternal age (years) & $30.84 \pm 4.05$ & $31.47 \pm 4.17$ & 0.007 \\
Maternal weight $(\mathrm{Kg})$ & $52.63 \pm 6.73$ & $52.10 \pm 6.79$ & 0.22 \\
Body Mass Index (kg/m²) & $20.77 \pm 2.79$ & $20.73 \pm 3.18$ & 0.81 \\
Nulliparous & $388(92.6)$ & $1244(93.1)$ & 0.74 \\
Gestational age (weeks) & $38.47 \pm 1.43$ & $38.32 \pm 1.50$ & 0.07 \\
Birthweight (g) & $3196.0 \pm 468.9$ & $3168.4 \pm 462.0$ & 0.29 \\
Birthweight z-score & $0.11 \pm 1.02$ & $0.11 \pm 0.99$ & 0.10 \\
Low Birthweight (<2500 g) & $21(5.0)$ & $68(5.1)$ & 1.00 \\
Macrosomia (>4000 g) & $23(5.5)$ & $49(3.7)$ & 0.12 \\
Boys & $236(56.3)$ & $700(52.3)$ & 0.16 \\
Preterm birth & $27(6.4)$ & $90(6.7)$ & 0.91 \\
Small for gestational age (SGA) & $54(12.9)$ & $153(11.4)$ & 0.44 \\
Large for gestational age (LGA) & $21(5.0)$ & $63(4.7)$ & 0.80 \\
\hline
\end{tabular}

Data are presented as number (\%) or mean \pm SD

the fresh embryo transfer group $(30.8 \pm 4.1$ years versus $31.5 \pm 4.2$ years, $p<0.05)$. GnRH agonist long protocol dominated our ovarian stimulation protocol with $97.3 \%$ in Vitrolife group and $96.4 \%$ in Sage group, while the percentages of $\mathrm{GnRH}$ antagonist were only 2.6 vs $3.3 \%$, $p>0.05$. The average numbers of oocytes collected were $12.4 \pm 5.8$ and $12.07 \pm 6.1$ respectively. IVF/ICSI fertilization rates were comparable,74.0/81.7 \% in Vitrolife versus $75.6 / 81.8 \%$ in SAGE with similar numbers of embryos per patient $(5.2 \pm 0.1$ verse $5.0 \pm 0.2, p>0.05)$.

Table 2 Patients' characteristics and neonatal outcome after frozen-thawed cleavage-stage embryo transfer

\begin{tabular}{llll}
\hline & $\begin{array}{l}\text { Vitrolife } \\
(n=202)\end{array}$ & $\begin{array}{l}\text { SAGE } \\
(n=413)\end{array}$ & $p$ \\
\hline Maternal age (years) & $30.07 \pm 3.79$ & $30.64 \pm 3.86$ & 0.08 \\
Maternal weight (Kg) & $52.58 \pm 7.56$ & $52.24 \pm 7.00$ & 0.57 \\
Body Mass Index (kg/m²) & $20.35 \pm 3.66$ & $20.62 \pm 2.82$ & 0.32 \\
Nulliparous & $186(92.1)$ & $383(92.7)$ & 0.74 \\
Gestational age (weeks) & $38.68 \pm 1.34$ & $38.39 \pm 1.59$ & 0.03 \\
Birthweight (g) & $3300.6 \pm 441.3$ & $3256.0 \pm 466.7$ & 0.27 \\
Birthweight z-score & $0.30 \pm 0.99$ & $0.29 \pm 0.97$ & 0.96 \\
Low Birthweight (<2500 g) & $7(3.6)$ & $15(3.7)$ & 1.00 \\
Macrosomia (>4000 g) & $12(6.2)$ & $16(4.0)$ & 0.23 \\
Boys & $105(52)$ & $205(49.6)$ & 0.61 \\
Preterm birth & $8(4.3)$ & $23(6.0)$ & 0.38 \\
Small for gestational age (SGA) & $15(8.2)$ & $29(7.6)$ & 0.80 \\
Large for gestational age (LGA) & $11(6.0)$ & $23(6.1)$ & 0.33 \\
\hline Data are presented as number (\%) & mean 50 & & \\
\hline
\end{tabular}

Data are presented as number (\%) or mean \pm SD 


\section{Perinatal outcome}

The birth outcomes are shown in Tables 1 and 2. For fresh cleavage embryo transfer, the average weight of newborns was $3196.0 \pm 468.9 \mathrm{~g}$ in Vitrolife group and 3168.4 \pm $462.0 \mathrm{~g}$ in SAGE. For cryopreserved cleavage-stage embryo transfer, the mean birthweight was $3300.6 \pm 441.3 \mathrm{~g}$ and $3256.0 \pm 466.7 \mathrm{~g}$ in Vitrolife and SAGE, respectively.

Z-score values were used to further explore birthweight differences while adjusting for infant gender and gestational age. Similar to absolute birthweight, there was no significant association between the z-score values and the type of embryo culture medium.

Of the pregnancies resulting in a singleton live birth, no differences were found with respect to the rate of SGA and LGA, as well as the LBW and macrosomia (Tables 1 and 2). Gestational age was found to be slightly but significantly higher in frozen cleavage embryos cultured in Vitrolife than in SAGE media $(38.68 \pm 1.34$ weeks versus $38.39 \pm 1.59$ weeks, $p<0.05$ ).

Finally, multiple linear regression was used to determine the relationship between culture medium and birthweight, gestational age, infant gender, type of culture media, maternal age, maternal weight, fertilization method, fresh or frozen-thawed embryo transfer. Maternal weight, gestational age, male sex and frozen-thawed embryo transfer were found to correlate with birthweight positively (Table 3).

\section{Survivors of a vanished co-twin}

The 2370 singleton newborns included 239 survivors of a vanished co-twin, 10 survivors of a vanished triplet and 2121 primary singletons. Among the 239 survivors of a vanishing co-twin, 209 babies were from fresh embryo transfer and 30 were born after frozen embryo transfer. The proportion of survivors in Vitrolife and in SAGE were similar at 12.2 versus $11.8 \%$ in fresh embryo transfer. While in frozen embryo cohort, singleton survivors cultured in Vitrolife and in SAGE were in the proportion of 3.4 versus $5.5 \%$, which makes no statistical difference. Furthermore, considering the gestational age at

Table 3 Results of multiple regression analysis between birthweight and confounding factors among live born singletons

\begin{tabular}{lrrl}
\hline & $\beta$ & \multicolumn{1}{l}{ t } & $p$ \\
\hline Maternal age (per year) & -0.008 & -0.45 & 0.65 \\
Maternal weight (per kg) & 0.211 & 11.77 & $<0.001$ \\
Frozen thawed embryo (versus fresh embryo) & 0.079 & 4.25 & $<0.001$ \\
ICSI (versus IVF) & 0.009 & 0.48 & 0.63 \\
SAGE (versus Vitrolife) & -0.004 & -0.24 & 0.81 \\
Gestational age & 0.490 & 27.38 & $<0.001$ \\
Gender (male versus female) & 0.110 & 6.33 & $<0.001$ \\
\hline$\beta$
\end{tabular}

$\beta$ is the regression coefficient the vanishing time, the proportions of early, intermediate and late survivors from each culture media were also comparable (Table 4).

\section{Discussion}

The main finding of this retrospective study was that the type of culture medium did not significantly affect the birthweight in singletons after either fresh or frozenthawed cleavage-stage embryo transfer. The sex ratio, rate of SGA and LGA as well as the rate of LBW and macrosomia were all comparable between the two culture media.

Culture medium is essential for embryo development in IVF. However, the types and concentrations of nutrients contained in the culture medium vary amongst different brands [20]. Nowadays, seven types of culture media are commercially available [21]. Two are single-step culture media which include IVF Online and Irvine Scientific and five are sequential culture media including Cook Medical, In Vitro Care, Origio, SAGE and Vitrolife.

Increasing evidence indicated that embryo culture may adversely affect the developmental potential and overall health of the embryo. In animal experiments, embryo culture in commercial media systems were proven to result in imprinted methylation loss compared to in vivoderived embryos [22]. Blastocyst and fetal development rates across different culture media showed profound variation in mouse zygotes [23]. Yet the results of mouse embryo responding to human media cannot be transferable directly to human development due to the physiological differences between oocytes from the two distinct species.

As a result, many researchers have started to study the influence of culture media on neonatal birthweight. Dumoulin et al. [24] found that singletons conceived after IVF with fresh cleavage Day $2 / 3$ embryo transfer cultured in Vitrolife medium were heavier than those conceived after culturing in Cook medium. He further reinforced his conclusion in a small size cohort study of both fresh and frozen embryo transfer [18, 24]. Likewise, Eskildet al. [25] found that the birthweight as well as the placental weight differed between different culture media.

Table 4 Proportion of singletons originated from twin/triplet gestation and the gestational age at the time of vanishing

\begin{tabular}{|c|c|c|c|c|c|c|}
\hline & \multicolumn{3}{|l|}{ Fresh } & \multicolumn{3}{|c|}{ Frozen-thawed } \\
\hline & Vitrolife & SAGE & $p$ & Vitrolife & SAGE & $p$ \\
\hline Survivors from twins & $51(12.2)$ & $158(11.8)$ & 0.81 & $7(6.2)$ & $23(6.0)$ & 0.26 \\
\hline Survivors from triplets & $2(0.5)$ & $5(0.4)$ & 0.68 & $2(1.0)$ & $1(0.2)$ & 0.25 \\
\hline Early pregnancy loss & $28(6.6)$ & $66(4.9)$ & 0.17 & $2(1.0)$ & $9(2.4)$ & 0.5 \\
\hline $\begin{array}{l}\text { Intermediate } \\
\text { pregnancy loss }\end{array}$ & $22(5.3)$ & $86(6.4)$ & 0.42 & $5(2.2)$ & $14(3.4)$ & 0.06 \\
\hline Late pregnancy loss & $1(0.2)$ & $6(0.4)$ & 1.0 & 0 & 0 & N/A \\
\hline
\end{tabular}

Data are presented as number (\%)

N/A: Non-applicable 
However, recently studies comparing culture media found no significant differences in mean birthweight between singletons cultured in different media such as Global/ Vitrolife/G1.3media;G1.3/Global/G1.5media; Medicult/ Cook/Vitrolifemedia; Medicult/Cook and Vitrolife/Global/ SAGE media [10-12, 26, 27].

The major differences between Vitrolife and SAGE are the protein source and the ratio of lactate to pyruvate (L:P). Both HSA (Human serum albumin) and SSS (Synthetic serum supplement) were used in Vitrolife while only SSS was used in SAGE. Vitrolife has higher concentration of lactate and higher ratio of lactate to pyruvate, both of which are primary energy substrates for early embryos. Further, SAGE were found additional electrolytes and metals such as aluminum and manganese which were not listed as media components [21]. More surprisingly, most commercial embryo culture media including Vitrolife and Cook medical were proved containing a large variety of non-declared protein which could potentially influence embryonic development, gestational age and birthweight [28].

SAGE is mainly used in reproductive centers of the US and Asia. The singleton birthweight was reported vary between 3020 and $3341 \mathrm{~g}$ in frozen cleavage embryo transfer and 2940 to $3350 \mathrm{~g}$ after transferring fresh embryo cultured in SAGE [12, 29]. Vitrolife is, in contrast, more widely used in Europe and the reported birthweight varied between 2942 and $3453 \mathrm{~g}$ after Day 3 fresh embryo transfer and was reported as $3394 \mathrm{~g}$ in frozen-thawed group $[8,9]$.

It would therefore be interesting to compare the perinatal outcome of these two culture media in the same population as well as the same laboratory condition. To our knowledge, there has been only one study published comparing the effects of SAGE and Vitrolife media on the birthweight of neonates after fresh cleavage embryo transfer. It was reported that no difference was found between the mean birthweight, with $3246.10 \pm 220.6 \mathrm{~g}$ in 792 Vitrolife babies and $3293.9 \pm 262.6 \mathrm{~g}$ in 235 SAGE newborns [11]. Our results were consistent with them but with a lower mean birthweight. The birthweight was $3196.0 \pm 468.9 \mathrm{~g}$ on average in the Vitrolife group and $3168.4 \pm 462.0 \mathrm{~g}$ in the SAGE group. This small difference in birthweight might be due to the different population and life styles between the North and South of China. However, the previous study only compared the absolute birthweight without adjusting for gestational age and gender and lacked the associated data on frozen-thawed embryo transfer.

Okenet al. [14] found that gestational age and gender of the infant were the factors with the most influences on birthweight in singleton deliveries. Therefore accordingly, we used the adjusted $\mathrm{z}$-score $[25,30]$ to give a more accurate picture for birthweight comparisons. Furthermore, most of the previous studies have only focused on the birth outcomes from fresh Day2/3 embryo transfer and few of them had data from the frozen-thawed embryo transfer. Our results were in agreement with most of the previous researches but gave more information on the effects of culture media by comparing them in both fresh and frozen-thawed cleavage embryo transfer groups.

Consistent with other studies, our multiple regression analyses showed a significant positive correlation between maternal weight and birthweight as well as gestational age and birthweight [11]. Male sex and frozenthawed embryo transfer resulted in a higher birthweight than female sex and fresh embryo transfer which was also in agreement with other reports [31].

In our study, it is of interest that in the cleavage-stage cryopreservation group, embryos cultured in Vitrolife had significantly higher gestational age than those cultured in SAGE. The gestational age was $38.68 \pm 1.34$ weeks versus $38.39 \pm 1.59$ weeks, respectively. This result may be biased by the small sample size and the high elective cesarean rates. The cesarean rate in China was deeply influenced by the one child policy and other social factors. The cesarean rate was $75.8 \%$ in SAGE group and $78.7 \%$ in the other group.

As double embryo transfer is still the routine in China, vanishing twin effect [32] should be an indispensable phenomenon which has aroused a great interest these days. The prevalence rate of vanishing twin effect is among $10-12.5 \%$ of IVF singletons [33, 34]. There is a great concern that the survivor of the twin will have a poorer prognosis than primary singleton which has been called "the black sheep of the family". They were reported to suffer an increased risk of SGA, preterm birth, low birthweight and perinatal mortality $[35,36]$. Furthermore, the gestational age at the time of vanishing was inversely correlated with adverse neonatal outcome [32]. To eliminate the possible bias from the vanishing effect, proportions of survivors as well as their vanishing time in different culture media were calculated and no statistical differences were found.

The limitations of our study were obvious. Firstly, it was a retrospective study. The maternal age between the two media in the fresh embryo transfer group were significantly different. However, our multiple regression analysis confirmed that it was not a confounder for neonatal birthweight in this study. Secondly, only two culture media were tested. Thirdly, the impact of culture media had not been studied in the context of blastocyst transfer. With respect to the controversial effects of prolonged in vrito cultrue (blastocyst transfer) on birthweight [37-40], it is necessary to collect data on neonatal outcomes from blastocyst transfer in the further study. Fourthly, the number of cases in two culture media varied a lot in this study. The reason was that SAGE was the major culture medium 
Table 5 Cases distribution in two culture media

\begin{tabular}{llllll}
\hline \multirow{3}{*}{ Year (s) } & \multicolumn{2}{l}{ Cleavage } & & & \\
\cline { 2 - 5 } & $\begin{array}{l}\text { Vitrolife } \\
\text { (ET cases) }\end{array}$ & $\begin{array}{l}\text { SAGE } \\
\text { (ET cases) }\end{array}$ & $\begin{array}{l}\text { Vitrolife } \\
\text { (FET cases) }\end{array}$ & $\begin{array}{l}\text { SAGE } \\
\text { (FET cases) }\end{array}$ & $\begin{array}{l}\text { FET + ET } \\
\text { (cases) }\end{array}$ \\
\hline $2009-2010$ & 12 & 708 & 49 & 237 & 13 \\
2011 & 138 & 430 & 78 & 135 & 81 \\
2012 & 269 & 198 & 75 & 41 & 140 \\
\hline
\end{tabular}

$E T$ fresh embryo transfer

FET frozen-thawed embryo transfer

before 2012. However, since 2012, Vitrolife has gradually dominated the embryo culture media because it became the only medium to be permitted by Chinese Food and Drug Administration. The cases distribution was listed in detail (Table 5). However, these two culture media were used randomly during the study period and our embryo culture conditions as well as the technicians in our center remained the same. Last but not least, a follow up study is needed to explore the long term effects of culture media [41] and the potential epigenetic changes in human embryos that have been suspected lately [42].

\section{Conclusion}

In conclusion, commercially available types of culture media, Vitrolife and SAGE were not associated with birthweight of singleton newborns after fresh or frozenthawed cleavage-stage embryo transfer.

\begin{abstract}
Abbreviations
ART: Assisted reproductive technology; GnRH: Gonadotropin-releasing hormone; HAS: Human serum albumin; hCG: Human chorionic gonadotropin; HRT: Hormone replacement therapy; ICSI: Intracytoplasmic sperm injection; IVF: In vitro fertilization; LGA: Large for gestational age; SGA: Small for gestational age; SSS: Synthetic serum supplement
\end{abstract}

\section{Acknowledgements}

We thanked the two students Han Li and Simin Ruan for their great work on raw data collecting and the follow up of pregnancy outcome.

\section{Funding}

The study was funded by the Ministry of Health Public Welfare Scientific Research Special Fund (201402004), Science and Technology Program of Guangzhou, China (201300000097), Special Fund of Guangdong Provincial Key Laboratory of Reproductive (2012A061400003) and National Natural Science Foundation of China (81370765).

\section{Availability of data and materials}

The datasets during and/or analyzed during the current study were available from the corresponding author on reasonable request.

\section{Authors' contributions}

YWX and CQZ conceived and designed the study; FG, MFD and J.G coordinated data collection and analyzed the data; F.G performed the statistical analysis and drafted the final report. ZW and CHD participated in its design and coordination. All authors read and approved the final manuscript.

\section{Competing interests}

The authors declare that they have no competing interests.

\section{Ethics approval and consent to participate}

The research protocol was approved by Ethic Review Committee of the First Affiliated Hospital of SunYat-sen University and informed consents were obtained prior to data collection.

\section{Author details}

'Department of Obstetrics and Gynecology, Reproductive Medical Center, The First Affiliated Hospital of SunYat-senUniversity, No 58 Zhongshan Road 2, Yuexiu District, Guangzhou 510080, China. ${ }^{2}$ Key Laboratory of Reproductive Medicine of Guangdong Province, Guangzhou, China. ${ }^{3}$ Department of Obstetrics and Gynecology, The First Affiliated hospital of SunYat-senUniversity, No 58 Zhongshan Road 2, Yuexiu District, Guangzhou 510080, China.

Received: 19 January 2016 Accepted: 13 September 2016

Published online: 19 September 2016

\section{Reference0073}

1. Hansen M, Kurinczuk JJ, Bower C, Webb S. The risk of major birth defects after intracytoplasmic sperm injection and in vitro fertilization. N Engl J Med. 2002;346(10):725-30.

2. Helmerhorst FM, Perquin DA, Donker D, Keirse MJ. Perinatal outcome of singletons and twins after assisted conception: a systematic review of controlled studies. BMJ. 2004;328(7434):261.

3. Jackson RA, Gibson KA, Wu YW, Croughan MS. Perinatal outcomes in singletons following in vitro fertilization: a meta-analysis. Obstet Gynecol. 2004;103(3):551-63.

4. Pandey S, Shetty A, Hamilton M, Bhattacharya S, Maheshwari A. Obstetric and perinatal outcomes in singleton pregnancies resulting from IVF/ICSI: a systematic review and meta-analysis. Hum Reprod Update. 2012;18(5):485-503.

5. Schieve LA, Meikle SF, Ferre C, Peterson HB, Jeng G, Wilcox LS. Low and very low birth weight in infants conceived with use of assisted reproductive technology. N Engl J Med. 2002;346(10):731-7.

6. Sazonova A, Kallen K, Thurin-Kjellberg A, Wennerholm UB, Bergh C. Factors affecting obstetric outcome of singletons born after IVF. Human Reprod. 2011;26(10):2878-86

7. Pinborg A, Wennerholm UB, Romundstad LB, Loft A, Aittomaki K, Soderstrom-Anttila $V$, et al. Why do singletons conceived after assisted reproduction technology have adverse perinatal outcome? Systematic review and meta-analysis. Hum Reprod Update. 2013;19(2):87-104.

8. Dumoulin JC, Land JA, Van Montfoort AP, Nelissen EC, Coonen E, Derhaag $J G$, et al. Effect of in vitro culture of human embryos on birthweight of newborns. Human Reprod. 2010;25(3):605-12.

9. Nelissen EC, Van Montfoort AP, Coonen E, Derhaag JG, Geraedts JP, Smits $L$, et al. Further evidence that culture media affect perinatal outcome: findings after transfer of fresh and cryopreserved embryos. Human Reprod. 2012;27(7):1966-76.

10. Eaton JL, Lieberman ES, Stearns C, Chinchilla M, Racowsky C. Embryo culture media and neonatal birthweight following IVF. Human Reprod. 2012;27:375-9.

11. Lin S, Li M, Lian Y, Chen L, Liu P. No effect of embryo culture media on birthweight and length of newborns. Human Reprod. 2013;28(7):1762-7.

12. Vergouw CG, Kostelijk EH, Doejaaren E, Hompes PG, Lambalk CB, Schats $R$. The influence of the type of embryo culture medium on neonatal birthweight after single embryo transfer in IVF. Human Reprod. 2012;27(9):2619-26.

13. Alpha Scientists in Reproductive M, Embryology ESIGo. The Istanbul consensus workshop on embryo assessment: proceedings of an expert meeting. Human Reprod. 2011;26(6):1270-83.

14. Oken E, Kleinman KP, Rich-Edwards J, Gillman MW. A nearly continuous measure of birth weight for gestational age using a United States national reference. BMC Pediatr. 2003;3:6.

15. Coleman T, Cooper S, Thornton JG, Grainge MJ, Watts K, Britton J, et al. A randomized trial of nicotine-replacement therapy patches in pregnancy. N Engl J Med. 2012;366(9):808-18.

16. Griesinger G, Kolibianakis EM, Diedrich K, Ludwig M. Ovarian stimulation for IVF has no quantitative association with birthweight: a registry study. Human Reprod. 2008;23(11):2549-54.

17. Huang XY, Liu HL. The trend growth of fetal weight in an average of time for different gestational age and gender. Maternal Child Heath Care. 2011;30:4701-3. 
18. Chang AL, Hurwitz E, Miyamura J, Kaneshiro B, Sentell T. Maternal risk factors and perinatal outcomes among pacific islander groups in Hawaii: a retrospective cohort study using statewide hospital data. BMC Pregnancy and Childbirth. 2015:15:239.

19. Zhang WY. Appendix 3:neonatal birthweight by gestation. In: Zhang WY, editor. Chinese perinatology. 1st ed. China: People's Medical Publishing House; 2012. p. 1592-3.

20. Mantikou $E$, Youssef MA, van Wely M, van der Veen F, Al-Inany HG, Repping $S$, et al. Embryo culture media and IVF/ICSI success rates: a systematic review. Hum Reprod Update. 2013;19(3):210-20.

21. Morbeck DE, Krisher RL, Herrick JR, Baumann NA, Matern D, Moyer T. Composition of commercial media used for human embryo culture. Fertil Steril. 2014;102(3):759-66. e759.

22. Market-Velker BA, Fernandes AD, Mann MR. Side-by-side comparison of five commercial media systems in a mouse model: suboptimal in vitro culture interferes with imprint maintenance. Biol Reprod. 2010;83(6):938-50.

23. Schwarzer C, Esteves TC, Arauzo-Bravo MJ, Le Gac S, Nordhoff V, Schlatt S, et al. ART culture conditions change the probability of mouse embryo gestation through defined cellular and molecular responses. Human Reprod. 2012;27(9):2627-40.

24. Nelissen EC, Van Montfoort AP, Smits LJ, Menheere PP, Evers JL, Coonen E et al. IVF culture medium affects human intrauterine growth as early as the second trimester of pregnancy. Human Reprod. 2013;28(8):2067-74.

25. Eskild A, Monkerud L, Tanbo T. Birthweight and placental weight; do changes in culture media used for IVF matter? Comparisons with spontaneous pregnancies in the corresponding time periods. Human Reprod. 2013;28(12):3207-14

26. Carrasco B, Boada M, Rodriguez I, Coroleu B, Barri PN, Veiga A. Does culture medium influence offspring birth weight? Fertil Steril. 2013;100(5):1283-8.

27. Lemmen JG, Pinborg A, Rasmussen S, Ziebe S. Birthweight distribution in ART singletons resulting from embryo culture in two different culture media compared with the national population. Human Reprod. 2014;29(10):2326-32.

28. Dyrlund TF, Kirkegaard K, Poulsen ET, Sanggaard KW, Hindkjaer JJ, Kjems J, Enghild JJ, Ingerslev HJ. Unconditioned commercial embryo culture media contain a large variety of non-declared proteins: a comprehensive proteomics analysis. Human Reprod. 2014;29(11):2421-30.

29. Kato O, Kawasaki N, Bodri D, Kuroda T, Kawachiya S, Kato K, et al. Neonatal outcome and birth defects in 6623 singletons born following minimal ovarian stimulation and vitrified versus fresh single embryo transfer. Eur J Obstet Gynecol Reprod Biol. 2012;161(1):46-50.

30. Land JA. How should we report on perinatal outcome? Human Reprod. 2006;21(10):2638-9.

31. Hsieh TT, Liou JD, Hsu JJ, Lo LM, Chen SF, Hung TH. Advanced maternal age and adverse perinatal outcomes in an Asian population. Eur J Obstet Gynecol Reprod Biol. 2010;148(1):21-6.

32. Pinborg A, Lidegaard O, Freiesleben N, Andersen AN. Vanishing twins: a predictor of small-for-gestational age in IVF singletons. Human Reprod. 2007;22(10):2707-14

33. La Sala GB, Villani MT, Nicoli A, Gallinelli A, Nucera G, Blickstein I. Effect of the mode of assisted reproductive technology conception on obstetric outcomes for survivors of the vanishing twin syndrome. Fertil Steril. 2006;86(1):247-9.

34. Pinborg A, Lidegaard $\mathrm{O}$, la Cour Freiesleben $\mathrm{N}$, Andersen AN. Consequences of vanishing twins in IVF/ICSI pregnancies. Human Reprod. 2005:20(10):2821-9.

35. Luke B, Brown MB, Grainger DA, Stern JE, Klein N, Cedars MI. The effect of early fetal losses on twin assisted-conception pregnancy outcomes. Fertil Steril. 2009;91(6):2586-92.

36. Shebl O, Ebner T, Sommergruber M, Sir A, Tews G. Birth weight is lower for survivors of the vanishing twin syndrome: a case-control study. Fertil Steril. 2008;90(2):310-4.

37. Fernando D, Halliday $J$, Breheny S, Healy DL. Outcomes of singleton births after blastocyst versus nonblastocyst transfer in assisted reproductive technology. Fertil Steril. 2012;97(3):579-84.

38. Kausche A, Jones GM, Trounson AO, Figueiredo F, MacLachlan V, Lolatgis N. Sex ratio and birth weights of infants born as a result of blastocyst transfers compared with early cleavage stage embryo transfers. Fertil Steril. 2001;76(4):688-93.

39. Schwarzler P, Zech H, Auer M, Pfau K, Gobel G, Vanderzwalmen P, et al. Pregnancy outcome after blastocyst transfer as compared to early cleavage stage embryo transfer. Human Reprod. 2004;19(9):2097-102.
40. Zhu J, Lin S, Li M, Chen L, Lian Y, Liu P, et al. Effect of in vitro culture period on birthweight of singleton newborns. Human Reprod. 2014:29(3):448-54.

41. Kleijkers SH, van Montfoort AP, Smits LJ, Viechtbauer W, Roseboom TJ, Nelissen EC, et al. IVF culture medium affects post-natal weight in humans during the first 2 years of life. Human Reprod. 2014;29(4):661-9.

42. Kleijkers SH, Eijssen LM, Coonen E, Derhaag JG, Mantikou E, Jonker MJ, et al. Differences in gene expression profiles between human preimplantation embryos cultured in two different IVF culture media. Human Reprod. 2015:30(10):2303-11.

\section{Submit your next manuscript to BioMed Central and we will help you at every step:}

- We accept pre-submission inquiries

- Our selector tool helps you to find the most relevant journal

- We provide round the clock customer support

- Convenient online submission

- Thorough peer review

- Inclusion in PubMed and all major indexing services

- Maximum visibility for your research

Submit your manuscript at www.biomedcentral.com/submit
Biomed Central 